\title{
LINEAR VISCOELASTIC MATERIALS WITH ENVIRONMENTAL DEPENDENT PROPERTIES
}

\author{
D. C. STOUFFER $\dagger$ \\ University of Cincinnati, Cincinnati, Ohio 45221 , U.S.A. \\ and \\ A. S. WINEMAN $\ddagger$ \\ University of Michigan, Ann Arbor, Mich. 48104, U.S.A.
}

\begin{abstract}
This study expands on the linear theory of thermo-viscoelasticity to include a larger group of materials and environmental effects. The development rests on the observation that for many materials the creep curve which results from a test at one steady value of an environmental parameter can be mapped onto the creep curve at another value of the environmental parameter by: (1) displacing the curve along the logarithmic time axis, (2) scaling the initial elastic response, and (3) scaling the long term or residual response. A thickwall, environmental-dependent, incompressible, viscoelastic cylinder is analyzed for a material that can be modeled by the proposed mapping. It is found that this mapping can have a profound effect on the resulting stress distributions. Further, using the above mapping a constitutive relationship is developed for transient environmental fields.
\end{abstract}

\section{INTRODUCTION}

THE LINEAR theory of viscoelasticity has been formulated and applied to situations in which environmental factors such as temperature are assumed to be constant. However, the mechanical response of a viscoelastic material is sensitive to variations in such environmental factors as temperature, humidity and presence of a diffusant. The purpose of this study is to extend the linear theory of viscoelasticity to include the effects of a larger set of environmental factors on a larger class of materials. The study is based on the observation that for many materials creep curves which result from tests at various steady values of, say, humidity, have the same general shape. Thus, the development rests on the hypothesis that the response curve at one constant humidity value can be mapped onto the response curve at another constant humidity value by: (1) displacing the curve along the logarithmic time axis, (2) scaling the initial elastic response, and (3) scaling the long term or residual response. This mapping could be characterized as an extension of the "Thermorheologically Simple" theory for material response [1].

In 1960 Morland and Lee [2] extended the Thermorheologically Simple model for viscoelastic response to include time dependent temperature fields. In this study the work of Morland and Lee is carefully reviewed and a technique is developed to extend the above mapping to transient environmental fields.

In order to provide the proper framework for this study, the general constitutive law for linear, environmental dependent viscoelastic materials is developed in section 2 .

Section 3 introduces a mapping hypothesis which relates creep or relaxation functions to different steady, homogeneous environments. Restrictions on the mapping and its relation to experimental data are discussed.

In section 4 a thick wall, environmental-dependent viscoelastic cylinder is analyzed

$\uparrow$ Assistant Professor of Engineering Science.

$\$$ Associate Professor of Engineering Mechanics. 
for constant internal pressure history. The generalized mapping is used to characterize the material response and an exact solution is obtained. A parameterization study demonstrates that the generalized mapping has a profound effect on the resultant stress distribution.

In section 5 the basic ideas are developed to extend the steady state results to transient environmental fields. It is shown that the results are consistent with those given by Morland and Lee [2] for "Thermorheologically Simple" materials; but the final form of the constitutive law is more complicated due to the basic nature of the problem. In conclusion, section 6 , the model is stated to include both time and spatially dependent environmental fields.

One final comment should be made regarding this study. A phenomenological approach is used throughout. While it is recognized that environmental phenomena may effect the mechanical properties of the material, no attempt is made to correlate these phenomena to the molecular structure of the material.

\section{THE BASIC CONSTITUTIVE RELATIONSHIP}

Let $\sigma_{i j}(\mathbf{x}, t)$ and $\epsilon_{i j}(\mathbf{x}, t)$ be the components with respect to some Cartesian coordinate system of the stress and infinitesimal strain tensors of a particle occupying position $\mathbf{x}$ at time $t$ in body $B$, and denote them by $\sigma$ and $\epsilon$, respectively. Next, consider the environmental parameters that can influence the mechanical response of a material; for example, temperature, humidity, concentration, radiation intensity, etc. Let $\phi(\mathbf{x}, t)$ represent the set of all environmental properties of the particle at position $\mathrm{x}$ in body $B$ at time $t$. Assume these functions to be defined and continuous for $\mathbf{x}$ in $B$ and $t$ in the interval $[0, \infty)$.

It is possible to obtain an integral representation for the constitutive law if the stress $\sigma(t)$ is linear in the strain history, translation invariant, non-retroactive, and continuous. $\dagger$ The linearity property allows any strain history $\epsilon$ to be associated with each fixed environmental history. This implies that the constitutive law can be written as a Riemann-Stieltjes integral in a form similar to that obtained by Gurtin and Sternberg [3]. Thus assume

$$
\sigma_{i j}(t)=\int_{0^{-}}^{t} \epsilon_{k l}(t-\tau) \mathrm{d} G_{i j k l}[\tau, \underset{s=0}{t}(s)]
$$

Here $G_{i j k l}$ are components of a fourth order tensor valued functional which (1) have the symmetry properties $G_{i j k l}=G_{j i k l}=G_{i j k k}$, (2) are of bounded variation on every sub interval of $[-a, \infty)$ for some $a>0$, and (3) which vanish on $[-a, 0)$ and are continuous on the right in $[0, \infty)$. Because the limits of the integral are from $0^{-}$to $t$, terms can arise from a possible jump discontinuity in $G_{i j k l}$ at $\tau=0$, i.e., the initial elastic response is automatically included in (2.1).

The physical meaning of the function $G_{i j k l}$ in (2.1) can be explained by letting $\epsilon_{k l}(\tau)$ have one non-vanishing component which is a unit step strain history applied at time $t_{0}>0$. The corresponding stress history predicted by $(2.1)$ has the form

$$
\sigma(t)=G\left[t-t_{0} ; \underset{s=0}{t}(s)\right]
$$

†The properties are defined in section 2 of reference [3]. 
showing that $G$ is a stress relaxation function measured in the presence of an environmental history $\phi$ for the entire time interval $[0, t]$. Let us assume that the environmental history on the interval when $\epsilon=0$ does not affect later mechanical response. Then (2.2) can be rewritten as

$$
\sigma(t)=G\left[t-t_{0} ; \underset{s=t_{0}}{t}(s)\right]=G[\tau ; \underset{s=t-\tau}{t}(s)]
$$

where $\tau$ represents the time elapsed since the step strain history was applied. Combining (2.1) and (2.3), the constitutive equation reduces to

$$
\sigma_{i j}(t)=\int_{0^{-}}^{t} \epsilon_{k l}(t-\tau) \mathrm{d} G_{i j k l}[\tau, \underset{s=t-\tau}{t}(s)]
$$

Equation (2.4) can also be formally derived using (2.3) in the usual physical arguments associated with the construction of the Boltzmann superposition integral.

If $G_{i j k l}$ has a continuous first derivative for $\tau$ in $[0, \infty)$ equation (2.4) can be rewritten as a Riemann integral. Equation (2.4) can be further specialized by assuming that the material is isotropic. The tensorial relaxation functional can be replaced by two scalar functionals and the constitutive equation becomes

$$
\sigma_{\alpha}(t)=\epsilon_{\alpha}(t) G_{\alpha}[0, \phi(t)]+\int_{0^{+}}^{t} \epsilon_{\alpha}(t-\tau) \frac{\partial}{\partial \tau} G_{\alpha}[\tau, \underset{s=t-\tau}{t}(s)] \mathrm{d} \tau
$$

or integrating by parts gives

$$
\begin{gathered}
(\alpha=1,2) \\
\sigma_{\alpha}(t)=G_{\alpha}[t, \underset{s=0}{t}(s)] \epsilon_{\alpha}(0)+\int_{0^{+}}^{t} G_{\alpha}[t-\tau, \underset{s=\tau}{\phi(s)}] \frac{\partial}{\partial \tau} \epsilon_{\alpha}(\tau) \mathrm{d} \tau
\end{gathered}
$$

where, if $\alpha=1, G_{1}$ is the shear relaxation functional, $\sigma_{1}, \epsilon_{1}$ are corresponding components of the deviatoric tensors if $\alpha=2, G_{2}$ is the relaxation functional in dilatation, $\sigma_{2}=\sigma_{k k}, \epsilon_{2}=\epsilon_{k k}$.

In the preceding discussion it was assumed that the stress is determined by the strain and environmental histories. This assumption can be reversed and one could assume the strain is determined by the stress and environmental histories. This amounts to interchanging the roles of $\boldsymbol{\sigma}$ and $\epsilon$ in the preceding development. Thus, it follows immediately that

$$
\begin{gathered}
\epsilon_{\alpha}(t)=\sigma_{\alpha}(t) J_{\alpha}[0, \phi(t)]+\int_{0^{+}}^{t} \sigma_{\alpha}(t-\tau) \frac{\partial}{\partial \tau} J_{\alpha}[\tau, \underset{s=t-\tau}{\phi(s)}] \mathrm{d} \tau \\
(\alpha=1,2)
\end{gathered}
$$

or

$$
\epsilon_{\alpha}(t)=\sigma_{\alpha}(0) J_{\alpha}[t \underset{s=0}{\phi(s)}]+\int_{0^{+}}^{t} J_{\alpha}[t-\tau, \underset{s=\tau}{t}(s)] \frac{\partial}{\partial \tau} \sigma_{\alpha}(\tau) \mathrm{d} \tau .
$$

Here $J_{1}$ and $J_{2}$ are the creep functionals in shear and dilatation respectively, $\sigma_{\alpha}, \epsilon_{\alpha}$ are as defined above. 


\section{A REPRESENTATION OF THE RELAXATION FUNCTIONAL FOR STEADY ENVIRONMENTS}

The remainder of this study will be concerned with developing a representation for the manner in which the material response functionals depend on the environmental history. To understand the influence of an environment on a real material, it is necessary to look at available experimental data. Almost all of this data appears to be taken for environmental histories which are time and spatially constant. Thus, the representation in this study is based on a mapping of response functions corresponding to different constant environmental histories onto the response function corresponding to some reference environment. It is not expected that the mapping will be valid for all materials and environments, however, the range of applicability appears to be reasonably large.

Consider a typical experimental program for the determination of a material response functional. For convenience, let $G$ represent a one-dimensional relaxation function. Also let all environmental parameters be constant at some value $\Phi$, that is, $\Phi=\phi(t)$ for all $t$ in $(-\infty, \infty)$. Then, if a unit step strain history is applied at $t=0,(2.5)$ gives

$$
\sigma(t)=G(t, \Phi) \text {. }
$$

That is, the measured stress as a function of time is equal to the relaxation function. Also $G$ must be associated with the particular constant environmental history $\Phi$ during the test. A typical relaxation curve (see Fig. 1) is assumed to monotonically decrease from a defined initial modulus $G(0, \Phi)$ to a defined residual modulus $G(\infty, \Phi)$. Now let some environmental property, say temperature, be fixed at $\phi_{1}$ for $t$ in $(-\infty, \infty)$. If the new temperature state $\phi_{1}$ is not too different from $\Phi$, it is reasonable to assume the mechanical response will still be linear. Thus, the constitutive equations will still have the same form as (2.5) and a material response function can be determined experimentally at tha new environmental parameter $\phi_{1}$. In this manner a family of relaxation curves, as shown in Fig. 1, can be obtained. The notation $G\left(t, \phi_{p}\right)$ signifies the dependence of the relaxation function on an environmental history $\phi(t)=\phi_{p}$, which is constant throughout the body for the entire strain history.

Now assume the relaxation function $G(t, \Phi)$ can be mapped onto the relaxation

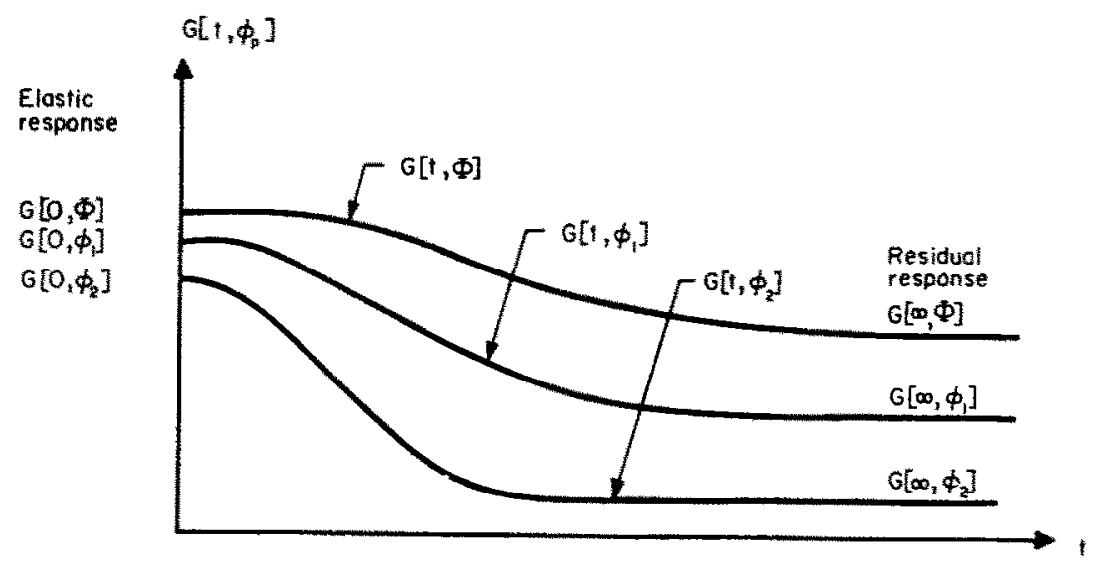

Fig. 1. Hypothetical dependence of a relaxation function on the variation of a particular environmental parameter. 
functions $G\left(t, \Phi_{p}\right)$ for each value of $\phi_{p}$. Further, this mapping has the form

$$
G\left(t, \phi_{p}\right)=\alpha\left(\phi_{p}\right) 1(t)+\beta\left(\phi_{p}\right) G\left[\gamma\left(\phi_{p}\right) t, \Phi\right],
$$

where the set of functions $\alpha, \beta$ and $\gamma$ depend only on $\phi_{p}$. The quantity $1(t)$ is the Heaviside unit step function which preserves the property that $G\left[t, \phi_{p}\right]$ vanishes on the negative time interval. The quantity $\Phi$ is the reference environmental constant for the mapping hypothesis.

The functions $\alpha, \beta$ and $\gamma$ are subject to a set of restrictions. The first is that the material response function $G\left(t, \phi_{p}\right)$ must reduce to the reference state response when $\phi_{p}=\Phi$. This requires that

$$
\begin{aligned}
& \alpha(\Phi)=0, \\
& \beta(\Phi)=1,
\end{aligned}
$$

and

$$
\gamma(\Phi)=1
$$

Next it is necessary to guarantee that the stress and strain always have the same sense. This requires that $G\left(t, \phi_{p}\right) \geqslant 0$ for all $t$ in $(-\infty, \infty)$. Thus the inequality

$$
\alpha\left(\phi_{p}\right)+\beta\left(\phi_{p}\right) G[t, \Phi] \geqslant 0, t \text { in }(0, \infty),
$$

places a limitation on the range of values that $\alpha\left(\phi_{p}\right)$ and $\beta\left(\phi_{p}\right)$ can assume.

Finally, in order that the sense of time be preserved, $\gamma\left(\phi_{p}\right)$ must satisfy

$$
\gamma\left(\phi_{p}\right)>0 .
$$

The assumption given by equation (3.2) states that the instantaneous (or elastic) response of the material for the environment at some value $\phi_{p}$ is $\left[\alpha\left(\phi_{p}\right)+\beta\left(\phi_{p}\right) G(0, \Phi)\right]$, and the long term (or residual) response is given by $\left[\alpha\left(\phi_{p}\right)+\beta\left(\phi_{p}\right) G(\infty, \Phi)\right]$. The function $\beta\left(\phi_{p}\right)$ scales the total amount of relaxation, and $\gamma\left(\phi_{p}\right)$ scales the relaxation time. Thus, analytically, (3.2) implies that changes in shape of the relaxation curves can be accounted for by scaling.

Similarly, it is convenient to introduce a creep function with the same structure as (3.2); i.e., assume the creep compliance function for some steady value of the environmental parameter $\phi_{p}$ is given by

$$
J\left(t, \phi_{p}\right)=\alpha\left(\phi_{p}\right) 1(t)+\hat{\beta}\left(\phi_{p}\right) J\left[\hat{\gamma}\left(\phi_{p}\right) t, \Phi\right] .
$$

In equation (3.6), $J(t, \phi)$ is the creep function in some reference state $\Phi ; \hat{\alpha}, \hat{\beta}$ and $\hat{\gamma}$ are mapping functions dependent only on $\phi_{p}$. Equation (3.6) is also subject to the restrictions of (3.3), (3.4) and (3.5). The relationships which exist between $\alpha, \beta, \gamma$ and $\hat{\alpha}, \hat{\beta}, \hat{\gamma}$ are given in the Appendix.

The mapping of (3.2) and (3.6) was motivated by reviewing much experimental data. The time shift factor is the same as that introduced by Schwarzl and Staverman [1] in connection with "Thermorheologically Simple" materials. The $\beta$ and $\hat{\beta}$ terms are of the 
type given in the W.L.F. equation (see [5]). The only new thing here is including the $\alpha$ and $\hat{\alpha}$ term. However, this does appear to significally increase the range of applicability of the mapping idea. A rather good correlation with experimental data was obtained for the influence of temperature on the mechanical response of nylon. Figure 2 demonstrates the effect of temperature on the time-dependent response of a nylon filament for total elongations up to seven per cent. An increase in temperature displaces the response curve upward. Selecting 21.5 degrees centigrade as the reference temperature, the temperature-dependent creep function (3.7) can be written as

$$
J(t, T)=0.0356(t-21 \cdot 5) 1(t)+j(t, 21 \cdot 5) .
$$

The data points calculated with this model are within 0.5 per cent of the experimental data. This accuracy verifies that a linear vertical shifting factor is reasonable for this application. Other places where the generalized mapping appears to work well are given in [5-9].

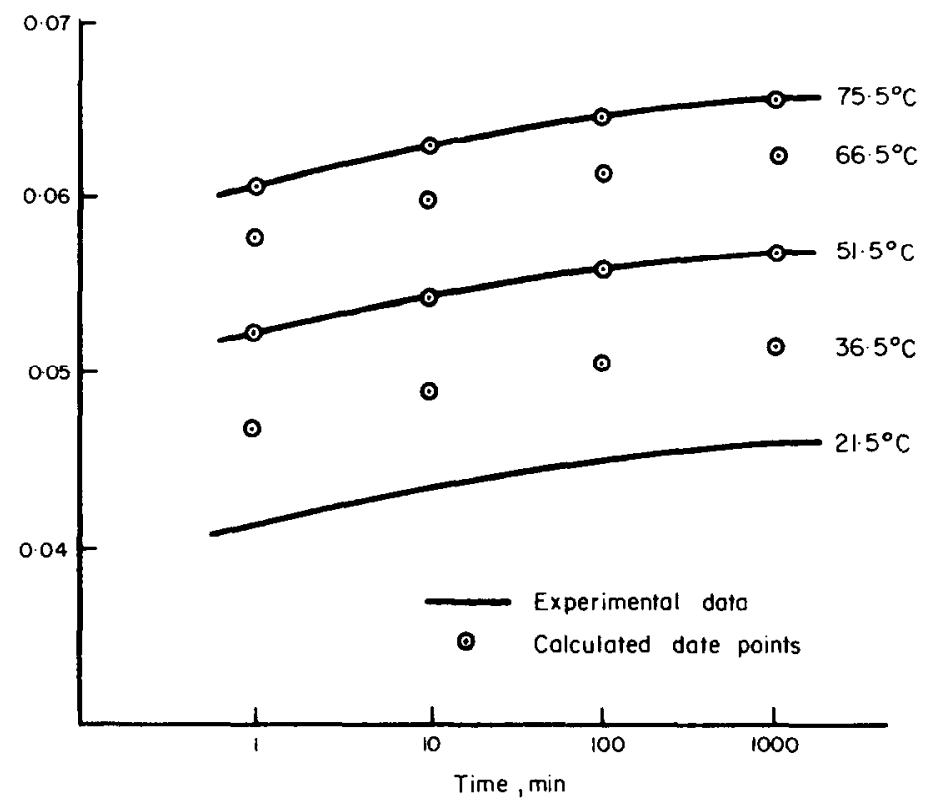

Fig. 2. Experimental and theoretical data for the creep or a nylon filament at several temperatures. (Experimental data from Leaderman H. Elastic and creep properties of filamentous materials and other high polymers 6.$)$

\section{INCOMPRESSIBLE THICKWALL CYLINDER IN PLANE STRAIN}

To investigate the implications of the assumed form of environmental dependence, a thickwall cylinder is examined in the state of plane strain. The problem is formulated for a linear incompressible viscoelastic material which has temperature-dependent shear response in creep and relaxation.

Let $(r, \theta, z)$ denote a generic point in a cylindrical polar coordinate system which is centered in body $B$. Body $B$ is a cylinder of inner radius $r=a$, outer radius $r=b$, and height $z= \pm Z$. The cylinder is analyzed for an internal pressure $P(t)=P I(t)$ where $P$ is a constant. The external pressure is taken as zero for all time. 
Due to the symmetry in the boundary conditions and body, the $(r, \theta, z)$ coordinate system coincides with the principal directions of the stress and strain tensors. Denote the components of the principal stress and strain tensors by $\left(\sigma_{r}, \sigma_{\theta}, \sigma_{z}\right)$ and $\left(\epsilon_{r}, \epsilon_{\theta}, \epsilon_{z}\right)$ respectively. Also, for this particular coordinate system and for the case of plane strain, all field quantities are independent of the $\theta$ and $z$ coordinates. Assume the displacement field is given by

$$
u=u_{r}(r, t) ; u_{\theta}=u_{z}=0 .
$$

Then the strain-displacement equations reduce to

$$
\epsilon_{r}(r, t)=u_{, r}(r, t) ; \quad \epsilon_{\theta}(r, t)=\frac{u(r, t)}{r} ; \epsilon_{z}=0 .
$$

In the absence of body forces the only non-trivial equilibrium equation is

$$
\sigma_{r, r}+\frac{\sigma_{r}-\sigma_{\theta}}{r}=0
$$

which must hold for each $t \geqslant 0$. Since the object of this study is to investigate the effect of temperature sensitive material response on the stress distribution, assume that the dilatational strain due to temperature is negligible. The stress-strain behavior in shear can be written as

$$
\sigma_{r}-\sigma_{\theta}=\int_{0^{-}}^{t}\left[\epsilon_{r}(r, t-\tau)-\epsilon_{\theta}(r, t-\tau)\right] \mathrm{d} G(\tau, r)
$$

where for convenience $G(t, r)$ denotes $G(t, T(r))$, the environmental dependent shear relaxation function.

To proceed, it is now necessary to pick a particular material or class of materials. Since the objective is to investigate the effect of the vertical scaling and shift factors of the mapping hypothesis introduced in section 3 on the mechanical field histories, let the relaxation response be given by

$$
G(t, \psi)=C_{1} \psi+\left(1+C_{2} \psi\right) G(t, \Phi),
$$

where $\psi=\phi_{p}-\Phi$ the deviation of some environmental parameter from the reference state $\Phi$. Further, assume mechanical response in the reference state is that of a three parameter solid, $\dagger$ then

$$
G(t, \Phi)=q_{0}+q_{1} \exp \left(-\frac{t}{p}\right)
$$

Combining the above gives the material response functional for a three parameter, viscoelastic solid subject to a constant environmental history as

$$
G[t, \psi]=C_{1} \psi+\left(1+C_{2} \psi\right)\left[q_{0}+q_{1} \exp \left(-\frac{t}{p}\right)\right]
$$

provided $t \geqslant 0$.

†See reference [10], p. 16. 
For convenience assume that the mechanical response of the three parameter solid is sensitive to temperature. Also for equation (4.5) to be valid the temperature must be constant for all time. Let $\phi=T(r)$ denote a temperature field which depends only on position and the thermal boundary conditions

$$
\begin{aligned}
& T(a)=T_{a} \text { for all } t \text { in }(-\infty, \infty), \\
& T(b)=T_{b} \text { for all } t \text { in }(-\infty, \infty)
\end{aligned}
$$

The solution of the stress boundary value problem together with the solution of Laplace's equation (reference [11]) for the temperature distribution gives

$$
\sigma_{r}(r, t)=P_{a}\left\{\frac{A(r)}{A(b)}+\frac{1}{A(b)}\left[B(r)-B(b) \frac{A(r)+B(r)}{A(b)+B(b)}\right] \exp \left[-\frac{A(b)}{A(b)+B(b)} \frac{t}{p}\right]-1\right\}
$$

and

$$
\sigma_{\theta}(r, t)=P_{a}\left\{\frac{A^{\prime}(r)}{A(b)}+\frac{1}{A(b)}\left[B^{\prime}(r)-B(b) \frac{A^{\prime}(r)+B^{\prime}(r)}{A(b)+B(b)} \exp \left[-\frac{A(b)}{A(b)+B(b)} \frac{t}{p}\right]-1\right\} .\right.
$$

The quantities $A, B, A^{\prime}$ and $B^{\prime}$ are given by

$$
\begin{aligned}
A(r) & =\frac{q_{0}\left(r^{2}-a^{2}\right)}{a^{2} r^{2}}+\frac{\left(C_{1}+C_{2} q_{0}\right)\left(T_{a}-T_{b}\right)}{a^{2} r^{2}}\left\{r^{2}-\frac{a^{2} \log (b / r)}{\log (b / a)}-\frac{\left(r^{2}-a^{2}\right)}{\log \left(b^{2} / a^{2}\right)}\right\}, \\
B(r) & =\frac{q_{1}\left(r^{2}-a^{2}\right)}{a^{2} r^{2}}+\frac{C_{2} q_{1}\left(T_{a}-T_{b}\right)}{a^{2} r^{2}}\left\{r^{2}-\frac{a^{2} \log (b / r)}{\log (b / a)}-\frac{\left(r^{2}-a^{2}\right)}{\log \left(b^{2} / a^{2}\right)}\right\}, \\
A^{\prime}(r) & =A(r)+F_{1}(r),
\end{aligned}
$$

and

$$
B^{\prime}(r)=B(r)+F_{2}(r) \text {. }
$$

$F_{1}(r)$ and $F_{2}(r)$ are given by

$$
F_{1}(r)=\frac{2}{r^{2}}\left[q_{0}+\left(C_{1}+C_{2} q_{0}\right)\left(T_{a}-T_{b}\right) \frac{\log (b / r)}{\log (b / a)}\right]
$$

and

$$
F_{2}(r)=\frac{2 q_{1}}{r^{2}}\left[1+C_{1}\left(T_{a}-T_{b}\right) \frac{\log (b / r)}{\log (b / a)}\right] .
$$

It is now possible to evaluate the effect of the mapping on the resulting stress distributions. For the special case when $T_{a}=T_{b}$ it follows that

$$
\begin{gathered}
A(r)=\frac{q_{0}\left(r^{2}-a^{2}\right)}{a^{2} r^{2}} ; B(r)=\frac{q_{1}\left(r^{2}-a^{2}\right)}{a^{2} r^{2}} \\
A^{\prime}(r)=\frac{q_{0}\left(r^{2}+a^{2}\right)}{a^{2} r^{2}} ; \quad B^{\prime}(r)=\frac{q_{1}\left(r^{2}+a^{2}\right)}{a^{2} r^{2}} .
\end{gathered}
$$

Then it is easy to show that the coefficients of the exponential terms in equations 
(4.9) vanish, and that the remaining terms reduce to the temporally constant stressstate given by the Lamé solution (reference [12]). Thus the temperature sensitive material properties introduce a time-dependent response.

Next consider the special case when $C_{1}=0$ in (4.5). This corresponds to scaling the initial response and residual response by the same factor. Then

$$
\frac{A(r)}{q_{0}}=\frac{B(r)}{q_{1}}
$$

and

$$
\frac{A^{\prime}(r)}{q_{0}}=\frac{B^{\prime}(r)}{q_{1}}
$$

This causes the exponential terms in (4.9) to vanish and the resulting stress field is again independent of time. The stress components for $C_{1}=0$ are

$$
\begin{gathered}
\sigma_{r}(r)=-P_{a}\left\{1-\frac{b^{2}\left[\left(r^{2}-a^{2}\right)+C_{2}\left(T_{a}-T_{b}\right)\left(r^{2}-a^{2} \frac{\log (b / r)}{\log (b / a)}-\frac{r^{2}-a^{2}}{\log \left(b^{2} / a^{2}\right)}\right)\right]}{r^{2}\left[\left(b^{2}-a^{2}\right)+C_{2}\left(T_{a}-T_{b}\right)\left(b^{2}-\frac{r^{2}-a^{2}}{\log \left(b^{2} / a^{2}\right)}\right)\right]}\right\} \\
\sigma_{\theta}(r)=-P_{a}\left\{1-\frac{b^{2}\left[\left(r^{2}+a^{2}\right)+C_{2}\left(T_{a}-T_{b}\right)\left(r^{2}+a^{2} \frac{\log (b / r)}{\log (b / a)}-\frac{r^{2}-a^{2}}{\log \left(b^{2} / a^{2}\right)}\right)\right]}{r^{2}\left[\left(b^{2}-a^{2}\right)+C_{2}\left(T_{a}-T_{b}\right)\left(b^{2}-\frac{r^{2}}{\log \left(b^{2} / a^{2}\right)}\right)\right]}\right\}
\end{gathered}
$$

To study the effect of temperature on the stress components, it is convenient to let

$$
a=1, b=2, \text { and } \xi_{2}=C_{2}\left(T_{a}-T_{b}\right)
$$

Figures 3 and 4 show the influence of $\xi_{2}$ on the radial and tangential stress components. The variable $\xi_{2}$ may be viewed in either of two ways. The first is to consider $C_{2}$ fixed, then $\xi_{2}$ represents the effect of temperature on the response of some particular material. The second approach is to hold $\left(T_{a}-T_{b}\right)$ fixed, then $\xi_{2}$ shows the response of a general class of materials which exhibit vertical scaling.

As shown in Figs. 3 and $4, \xi_{2}$ has very little influence on the radial stress; but, the influence on the tangential stress is quite pronounced. For a given material $\left(C_{2}\right.$ held fixed) the curves indicate that as $T_{a}-T_{b}$ increases, the magnitude of the stress component increases on the inner boundary and decreases on the outer boundary. If $T_{a}-T_{b}$ is held fixed, then as $\xi_{2}$ increases the magnitude of the stress components increase at $r=a$. Note that the effect of the vertical scaling causes a significant deviation from the elastic solution which corresponds to $\xi_{2}=0$.

Consider next the material response when $C_{1} \neq 0$ and $C_{2}=0$. This corresponds to changing the initial and residual response moduli by the same additive constant. Under these circumstances the equations for the stress-state retain the same basic form as 


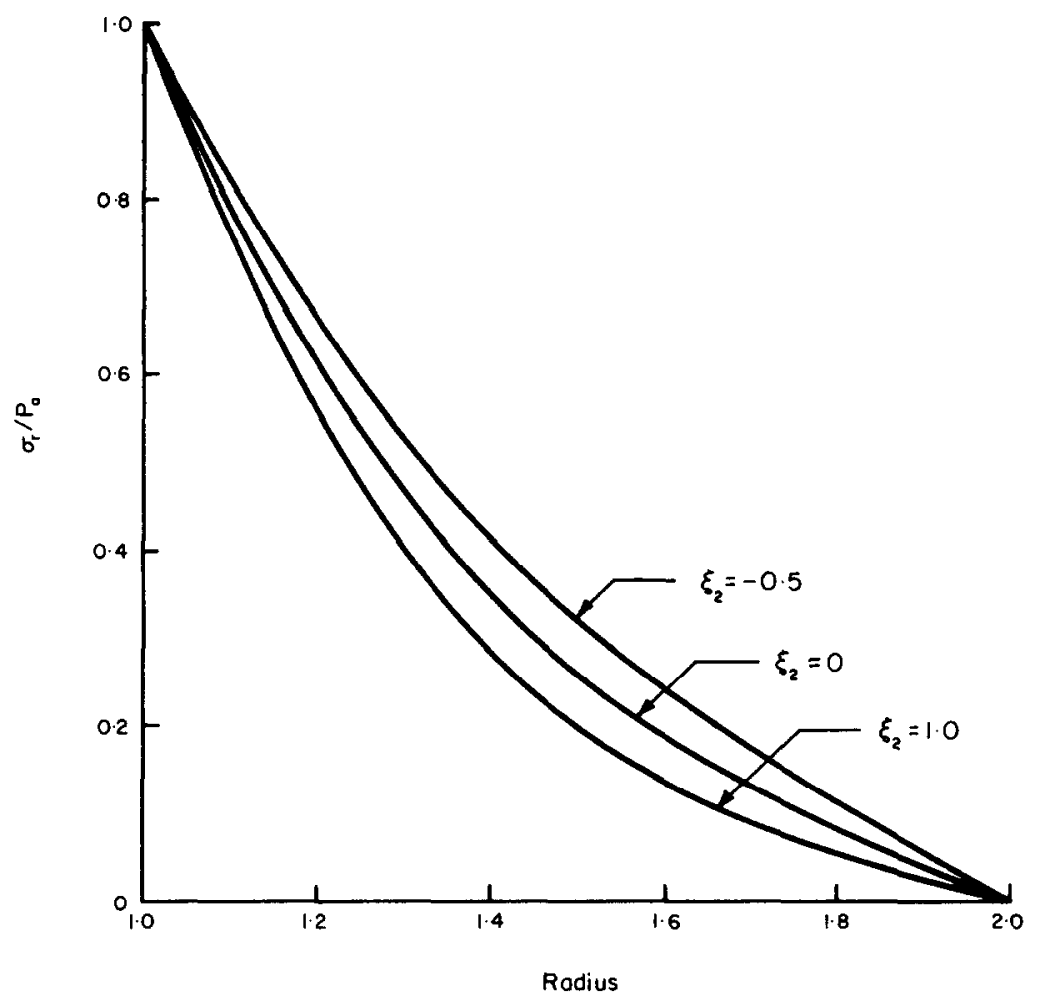

Fig. 3. Influence of $\xi_{2}$ on the radial stress component when $C_{1}=0$.

given in equation (4.9). Thus it is the presence of $C_{1}$ that introduces the exponential time term. For both stress components, the time independent behavior, as well as the rate and amount of viscoelastic response, all depend on $C_{1}\left(T_{a}-T_{b}\right)$.

For the purpose of studying the influence of the temperature term $C_{1}\left(T_{a}-T_{b}\right)$ on the stress histories, again set

$$
a=1, b=2, q_{0}=q_{1}=\frac{1}{2},
$$

and

$$
\xi_{1}=C_{1}\left(T_{u}-T_{b}\right)
$$

Figures 5 and 6 show the result of introducing (4.13) into (4.9) and evaluating $\sigma_{\theta}(r, t)$ at $r=a$ and $r=b$. As $\xi_{1}$ increases the general long time trend is to increase the magnitude of the stress components on the inner boundary and decrease the stress on the outer boundary. The relaxation time does not appear to be altered significantly by changes in $\xi_{1}$.

\section{REPRESENTATION FOR TRANSIENT ENVIRONMENTAL FIELDS}

Recall that $G\left[t, \phi_{p}\right]$ is the one-dimensional relaxation response for a material in a temporally constant environment $\phi_{p}$. The relaxation functional which depends on a transient environmental history is given by $G\left[t-t_{0}, \underset{s=t}{t}(s)\right]$, for a unit step strain applied 


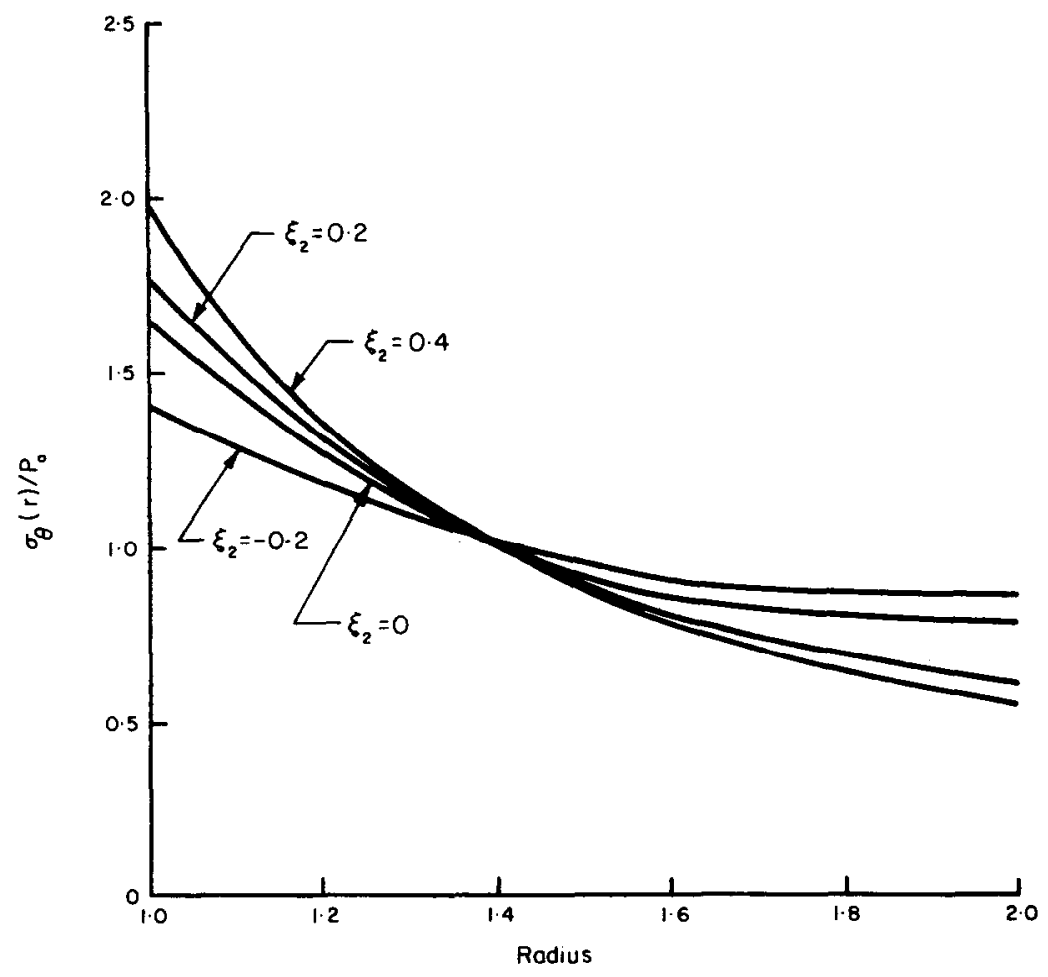

Fig. 4. Influence of $\xi_{2}$ on the tangential stress component when $C_{1}=0$.

a time $t_{0}$. For the development in this section, it is convenient to let $t_{0}=0$. The results for the more general case will be stated later.

The expansion to transient environments rest on the assumption that the amount of change in the relaxation function $\Delta G$ in the infinitesimal interval of time $[t, t+\Delta t]$ depends only on a representative value of the environment in that interval of time. The environmental history on $[0, t]$ has no influence on the stress in the time interval $[t, t+\Delta t]$ other than to determine the stress at time $t$. Furthermore, environmental changes cannot reverse the relaxation process; they can only vary the rate of relaxation. Although the validity of this assumption must be verified experimentally, it seems reasonable that it be at least approximately true for slowly varying environments.

As a consequence of this assumption $\Delta G$, which depends only on $G\left(t, \phi_{p}\right)$, can be ultimately expressed in terms of $G(t, \Phi)$. The problem then reduces to expressing $G[t, \underset{s}{\phi(s)}]$ in terms of $\alpha, \beta, \gamma$ and $G(t, \Phi)$. In order to examine the underlying aspects of this problem let the environmental history $\phi(t)$ be partitioned into $N$ sub-intervals. Let $\Delta t_{i}$ be the typical time interval $\left[t_{i-1}, t_{i}\right]$ and let $\phi_{i}$ be a representative value of the environmental history in the interval. Denote the value of $\phi(t)$ at $t=0$ by $\phi_{0}$. Now at $t=0$ we have

$$
G[\underset{s=0}{0, \stackrel{0}{\phi})}]=\alpha\left(\phi_{0}\right)+\beta\left(\phi_{0}\right) G(0, \Phi) \equiv \stackrel{\circ}{G}
$$




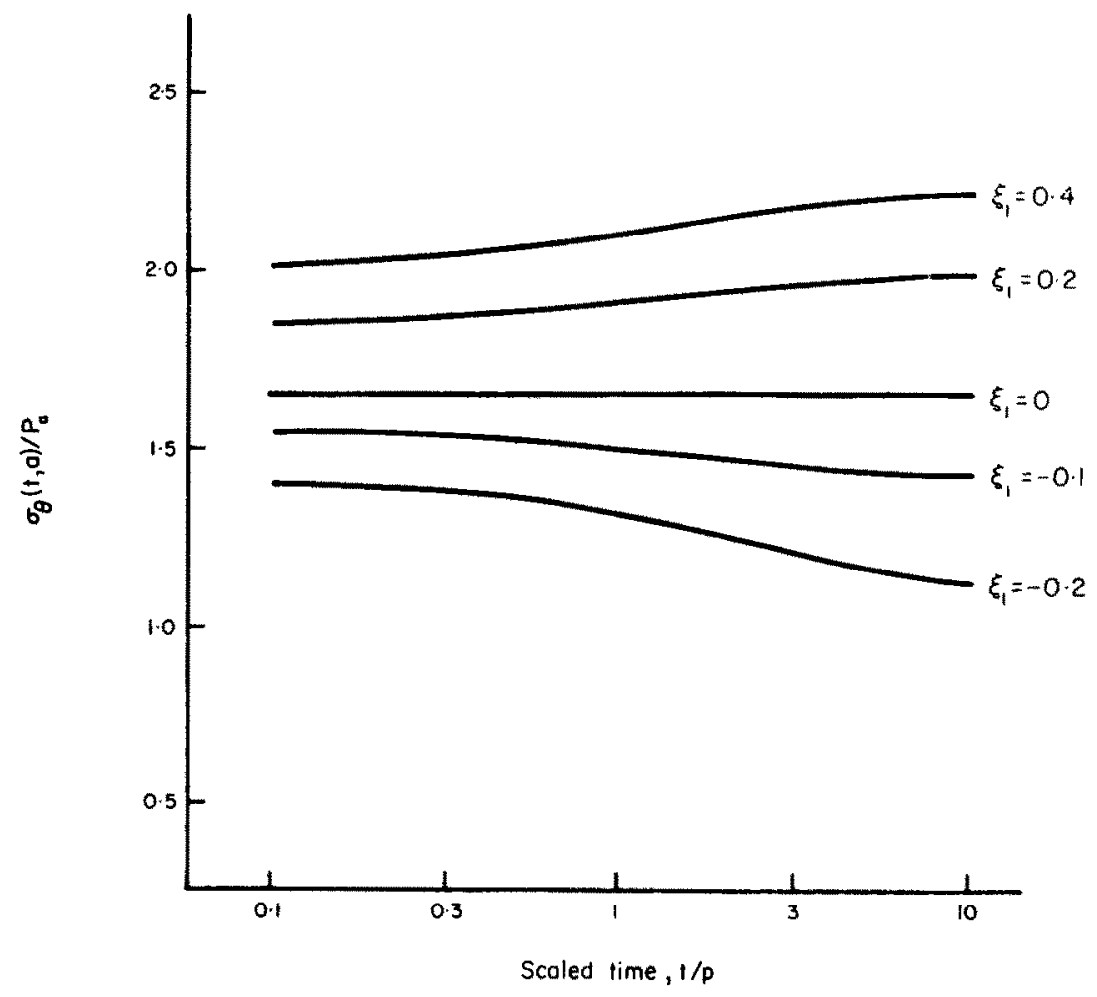

Fig. 5. Influence of $\xi_{1}$ on the tangential stress history at the inner boundary $r=a$ when $C_{2}=0$.

Since $\phi_{0}$ is the initial value of the environmental history, then (5.1) follows from equation (3.2).

During the first interval of time $\left(0, \Delta t_{1}\right]$, the relaxation functional will change an amount $\Delta G_{1}$ from the initial response. Hence, we can write

$$
G\left[\Delta t_{1}, \phi \underset{s=0}{\Delta t_{1}}(s)\right]=\stackrel{\odot}{G}+\Delta G_{1}
$$

The incremental change $\Delta G_{1}$ is comprised of two parts. The first part, corresponding to the first term in (3.2), is a vertical shift in the relaxation curve due to a change of $\phi$ from $\phi_{0}$ to $\phi_{1}$. This is motivated by treating the term $\alpha(\phi) 1(t)$ as an environmental dependent elastic modulus. The second part, corresponding to the second term (3.2), is the montonic decay of stress that occurs in the time interval $\left(0, \Delta t_{1}\right]$ at the environmental value $\phi_{1}$. That is,

$$
\Delta G_{1}=\left[\alpha\left(\phi_{1}\right)-\alpha\left(\phi_{0}\right)\right]+\beta\left(\phi_{1}\right)\left\{G\left[\gamma\left(\phi_{1}\right) \Delta t_{1}, \Phi\right]-G[0, \Phi]\right\} .
$$

This means when $\alpha=0$ and $\beta=1$ the amount of relaxation $\Delta G_{1}$ that occurs in the time interval $\left(0, \Delta t_{1}\right]$ for the environmental state $\phi_{1}$ will require a time interval $\gamma\left(\phi_{1}\right) \Delta t_{1}$ for the reference state $\Phi$ (see Fig. 7). Note in this case $\Delta G_{1}$ could be positive or 


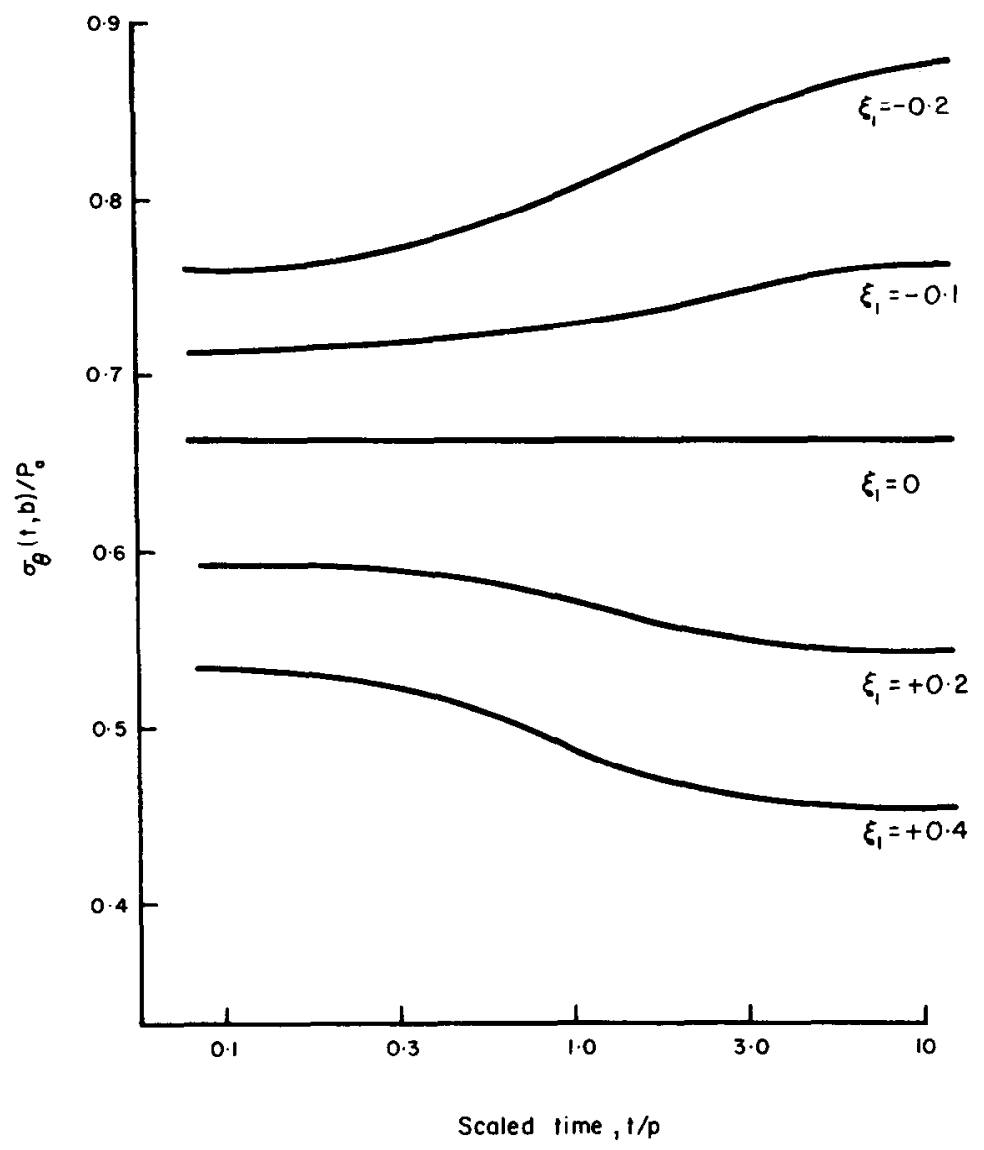

Fig. 6. Influence of $\xi_{1}$ on the tangential stress history at the outer boundary $r=b$ when $C_{2}=0$.

negative depending on the value of $\Delta \alpha_{1}$, and hence, $G[t, \underset{s=0}{t}(s)]$ is not required to be a monotonically decreasing function. Denoting

$$
\Delta \alpha_{i}=\alpha\left(\phi_{i}\right)-\alpha\left(\phi_{i-1}\right),
$$

the total response after $\Delta t_{1}$ is

$$
\begin{aligned}
\left.G\left[\Delta t_{1}, \underset{s=0}{\Delta t_{1}}\right)\right] & =\stackrel{\circ}{G}+\Delta G_{1} \\
= & \alpha\left(\phi_{0}\right)+\Delta \alpha_{1}+\beta\left(\phi_{1}\right) G\left[\gamma\left(\phi_{1}\right) \Delta t_{1}, \Phi\right]-G(0, \Phi)\left\{\beta\left(\phi_{1}\right)-\beta\left(\phi_{0}\right)\right\} .
\end{aligned}
$$

In the next time interval $\left(\Delta t_{1}, \Delta t_{1}+\Delta t_{2}\right], \phi$ takes on the representative value $\phi_{2}$. Then

$$
G\left[\Delta t_{1}+\Delta t_{2}, \stackrel{\Delta t_{1}+\Delta t_{2}}{\phi}(s)=\stackrel{\circ}{g}\right)=\Delta G_{1}+\Delta G_{2}
$$




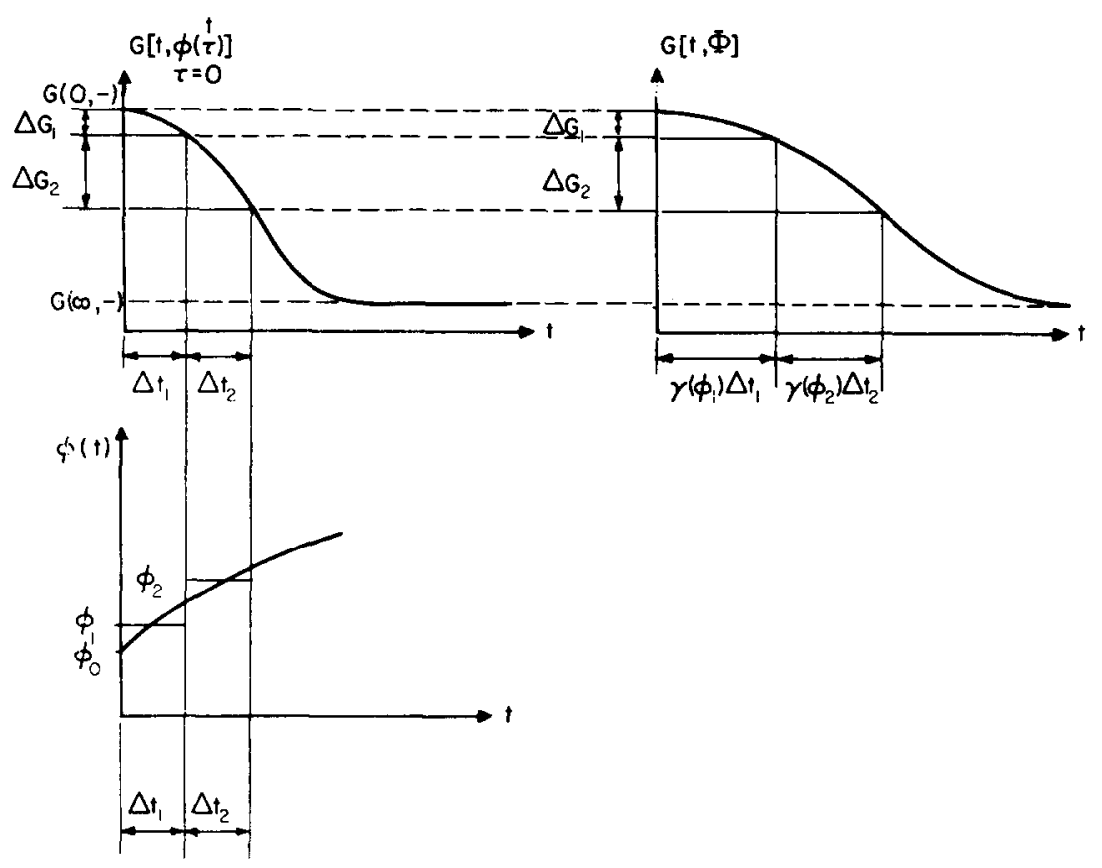

Fig. 7. Mapping of the time coordinate for a "Thermorheologically Simple" material.

where $\Delta G_{2}$ depends on $G\left(t, \phi_{2}\right)$. To find $\Delta G_{2}$, note the following consequence of (3.2). The monotonic relaxation at $\phi_{2}$ during time interval $\Delta t_{2}$ is $\beta\left(\phi_{2}\right)$ times the change in $G$ at $\Phi$ during the time interval $\gamma\left(\phi_{2}\right) \Delta t$, and is independent of when the interval begins. Combining this relaxation increment with the vertical shift due to a change in $\alpha$ gives

$$
\Delta G_{2}=\Delta \alpha_{2}+\beta\left(\phi_{2}\right)\left\{G\left[\gamma\left(\phi_{1}\right) \Delta t_{1}+\gamma\left(\phi_{2}\right) \Delta t_{2}, \Phi\right]-G\left[\gamma\left(\phi_{1}\right) \Delta t_{1}, \Phi\right]\right\}
$$

Substitution of (5.3) and (5.6) into (5.5) gives

$$
\begin{aligned}
& G\left[\Delta t_{1}+\Delta t_{2}, \underset{s=0}{\Delta t^{1}+\Delta t^{2}}\right]=\alpha\left(\phi_{0}\right)+\Delta \alpha_{2}+\beta\left(\phi_{2}\right) G\left[\gamma\left(\phi_{1}\right) \Delta t_{1}+\gamma\left(\phi_{2}\right) \Delta t_{2}, \Phi\right] \\
& -G(0, \Phi)\left\{\beta\left(\phi_{1}\right)-\beta\left(\phi_{0}\right)\right\}-G\left[\gamma\left(\phi_{1}\right) \Delta t_{1}, \Phi\right]\left\{\beta\left(\phi_{2}\right)-\beta\left(\phi_{1}\right)\right\} .
\end{aligned}
$$

Hence, after some time $t=\sum_{i=1}^{N} \Delta t_{i}$, the relaxation functional can be written as

$$
\begin{aligned}
& G[\underset{t, \phi(s)}{t}]=\alpha\left(\phi_{0}\right)+\sum_{i=1}^{N} \Delta \alpha_{i}+\beta[\phi(t)] G\left[\sum_{i=1}^{N} \gamma\left(\phi_{i}\right) \Delta t_{i}, \Phi\right] \\
& -\sum_{i=1}^{N} G\left[\sum_{p=0}^{i=1} \gamma\left(\phi_{p}\right) \Delta t_{p}, \Phi\right]\left\{\beta\left(\phi_{i}\right)-\beta\left(\phi_{i-1}\right)\right\} .
\end{aligned}
$$


Letting $N \rightarrow \infty$ for a fixed time interval $[0, t]$, equation (5.8) can be written as

$$
\begin{aligned}
G[t, \underset{s=0}{t}(s)]= & \alpha[\phi(t)]+\beta[\phi(t)] G\left[\int_{0^{+}}^{t} \gamma[\phi(\tau)] \mathrm{d} \tau, \Phi\right] \\
& -\int_{0^{+}}^{t} G\left[\int_{0^{+}}^{\tau} \gamma[\phi(\theta)] \mathrm{d} \theta, \Phi\right] \mathrm{d} \beta[\phi(\tau)] .
\end{aligned}
$$

Integrating equation (5.9) by parts yields

$$
\begin{aligned}
G\left[\begin{array}{l}
t, \phi(s) \\
s=0
\end{array}\right]= & \alpha[\phi(t)]+\beta[\phi(0) G[0, \Phi] \\
& +\int_{0^{+}}^{t} \beta[\phi(\tau)] \mathrm{d} G\left[\int_{0^{+}}^{\tau} \gamma[\phi(\theta)] \mathrm{d} \theta, \Phi\right] .
\end{aligned}
$$

Equation (5.10) can be arrived at by interchanging the summation order in equation (5.8).

This representation is consistent with the results of Morland and Lee[1] for "Thermorheologically Simple" materials. In this situation $\alpha=0$ and $\beta=1$, so (5.9) reduces to

$$
G[t \underset{s=0}{t}(s)]=G[\xi, \Phi]
$$

where

$$
\xi=\int_{0}^{t} \gamma[\phi(\theta)] \mathrm{d} \theta
$$

In equation (5.12) $\xi$ is the pseudo time $\dagger$ introduced by Morland and Lee and implies that the rate of relaxation can be accounted for by a one-to-one mapping of the time coordinate. $\neq$

The two forms of the relaxation function (5.9) and (5.10) are equivalent; however, depending upon the nature of the scaling functions $\alpha$ and $\beta$, equation (5.9) may be more useful in the solution of boundary value problems. In either case it will probably be necessary to use a numerical technique to determine the relaxation functional for a "real" material.

If equation (5.10) does not depend on $\beta$, then on setting $\beta[\phi(t)]=1(t)$, equation (5.10) reduces to

$$
\begin{aligned}
G[t, \underset{s=0}{t}(s)] & =\alpha[\phi(t)] l(t)+G\left[\int_{0}^{t} \gamma[\phi(\tau)] \mathrm{d} \tau, \Phi\right] \\
& =\alpha[\phi(t)] l(t)+G(\xi, \Phi),
\end{aligned}
$$

with $\xi$ defined by equation (5.12). For the special case of an elastic material $G[\xi, \Phi]$ is replaced by $E_{R} 1(t)$, where $E_{R}$ is the elastic modulus corrcsponding to the reference state

$\dagger$ For additional reference regarding the reduced time concept and its application, see references [13], [14] and [15].

$¥ I t$ was pointed out by Sternberg[13] that equations (3.3) and (3.5) are sufficient to guarantee that (5.12) has a unique inverse. 
$\Phi$. Then equation (5.13) becomes

$$
G[\underset{t}{t} \underset{s=0}{t}(s)]=E[\phi(t)] 1(t)
$$

Here $E[\phi(t)]$ is an environmental-dependent elastic modulus which depends only on the current value of the environment.

The material response functionals given by equations (5.9) and (5.10) can be modified to be compatible with the form of the relaxation functional used in equation (2.5), since this relaxation functional depends only on the environmental history in the time interval $\left[t_{0}, t\right]$. The representation of this functional can be obtained by carrying out the above derivation for the time interval $\left[t_{0}, t\right]$. Therefore, the relaxation functional of equation (2.5) is given by

$$
\begin{aligned}
G\left[t-t_{0}, \underset{s=t_{0}}{t}(s)\right]=\alpha[\phi(t)]+\beta[\phi(t)] G & {\left[\int_{t_{0}}^{t} \gamma[\phi(\theta)] \mathrm{d} \theta, \Phi\right] } \\
& -\int_{t_{0}}^{t} G\left[\int_{t_{0}}^{s} \gamma[\phi(\theta)] \mathrm{d} \theta, \Phi\right] \mathrm{d} \beta[\phi(s)],
\end{aligned}
$$

when $t>t_{0} ;$ or

$$
\begin{aligned}
G\left[t-t_{0}, \underset{s=t_{0}}{t}(s)\right]=\alpha[\phi(t)]+\beta\left[\phi\left(t_{0}\right)\right] G & {[0, \Phi] } \\
& +\int_{t_{0}}^{t} \beta[\phi(s)] \mathrm{d} G\left[\int_{t}^{s} \gamma[\phi(\theta)] \mathrm{d} \theta, \Phi\right] .
\end{aligned}
$$

As a final comment, note that the initial value of the relaxation functional, i.e., the initial elastic response, depends on the initial value of the environment $\phi_{0}$. The residual modulus, however, is not determined by the long time value of the environment but by the entire history $\phi(t)$. This can be seen from (5.6) in which the environmental history determines both the vertical shift $\Delta \alpha_{2}$ and the scaling $\beta(\phi)$ of the increment in $G(t, \Phi)$. This behavior is not given by the current Thermorheologically Simple model.

\section{TIME AND SPATIALLY-DEPENDENT ENVIRONMENTAL FIELDS}

For spatially variant environmental fields $\phi(t, \mathbf{x})$, the scaling factors $\alpha, \beta$ and $\gamma$ become functions of position and time. Then the response functional of the particle can be expressed by

$$
\begin{aligned}
G\left[t-t_{0}, \underset{s=t_{0}}{t(s)}\right]=\alpha[\phi(t, \mathbf{x})]+\beta\left[\phi\left(t_{0}, \mathbf{x}\right)\right] G(0, \Phi) & \\
& +\int_{t_{0}}^{t} \beta[\phi(s, \mathbf{x})] \mathrm{d} G\left[\int_{t_{0}}^{s} \gamma[(\theta, \mathbf{x})] \mathrm{d} \theta, \Phi\right] .
\end{aligned}
$$

The scaling factors $\alpha(\phi), \beta(\phi)$, and $\gamma(\phi)$, and the material response function in the reference state $G(t, \Phi)$ are known from an experimental program. The environmental history $\phi(t, \mathbf{x})$ can be found from the appropriate physical law and boundary conditions. Then equation (6.1) can be evaluated to give the material response function. 
The results of this study can be easily generalized to include three-dimensional stress and strain histories. For convenience with the notation, write the scaling factors as

$$
\begin{aligned}
\alpha(\mathbf{x}, t) & \equiv \alpha[\phi(\mathbf{x}, t)] 1(t), \\
\beta(\mathbf{x}, t) & \equiv \beta[\phi(\mathbf{x}, t)],
\end{aligned}
$$

and

$$
\gamma(\mathbf{x}, t) \equiv \gamma[\chi(x, t)] .
$$

Then the relaxation functionals in shear and dilatation can be written as

$$
\begin{aligned}
G_{i}\left[t-t_{0}, \phi \underset{s=t_{0}}{t}(\mathbf{x}, s)\right]=\alpha_{i}(\mathbf{x}, t)+\beta_{i}\left(\mathbf{x}, t_{0}\right) G_{i}(0, \Phi) & \\
& +\int_{t_{0}}^{t} \beta_{i}(\mathbf{x}, s) \mathrm{d} G_{i}\left[\int_{t_{0}}^{t} \gamma(\mathbf{x}, \theta) \mathrm{d} \theta, \Phi\right]
\end{aligned}
$$

for $i=1,2$.

If a creep response is considered, a similar development gives the isotropic creep functions in shear and dilatation as

$$
\begin{aligned}
J_{i}\left[t-t_{0}, \phi\left(\underset{s=t_{0}}{t}, s\right)\right]=\hat{\alpha}_{i}(\mathbf{x}, t)+\hat{\beta}_{i}\left(\mathbf{x}, t_{0}\right) J_{i}(0, \Phi) & \\
& +\int_{t_{0}}^{t} \hat{\beta}_{i}(\mathbf{x}, s) \mathrm{d} J_{i}\left[\int_{t_{0}}^{s} \gamma(\mathbf{x}, \theta) \mathrm{d} \theta, \Phi\right]
\end{aligned}
$$

for $i=1,2$.

This study was devoted to finding a specific representation for a class of environmental-dependent material response functions. It is desirable to correlate the results of this section with the constitutive models established in section 3 . In view of the complicated form of equations (6.3) and (6.4), it appears to be most convenient to use (6.3) when the relaxation law has form (2.5), and (6.4) in a creep law of form (2.6).

The mechanical response functions as given in (6.3) or (6.4) can readily be determined for a real material. The response function in the reference state and the scaling factors can be determined from an experimental program for the material being considered. The environmental history $\phi(\mathbf{x}, t)$ can be calculated from the appropriate physical law. Then performing the required integration yields either the creep or relaxation function of (6.3) or (6.4). Modern numerical techniques will allow the calculation of (6.3) or (6.4) with no difficulty for use in boundary value problems.

\section{REFERENCES}

[1] F. SCHWARZL and A. J. STAVERMAN, $J$, appl. Phys. 23.8, 838 (1952).

[2] L. W. MORLAND and E. H. LEE, Trans. Soc. Rheol. 4, 233 (1960).

[3] M. E. GURTIN and ELI STERNBERG, Arch. ration. Mech. Analysis 11.4, 291 (1962).

[4] J. D. FERRY, Viscoelastic Properties of Polymers. Wiley, New York (1961).

[5] H. LEADERMAN, Elastic and Creep Properties of Filamentous Materials and Other High Polymers. Textile Foundation (1943). 
[6] R. L. STEINBERGER, Creep in Cellulose Acetate Filaments. Textile Research (1936).

[7] P. S. THEOCARIS, Proc. of the Society of Experimental Stress Analysis 22.1 (1965).

[8] J. BISCHOFF, E. CATSIFF and A. V. TOBOLSKY, J.Am. chem. Soc. 74, 3378 (1952).

[9] M. HETENYI, Trans. Am. Soc. Mech. Engrs 60, A-149 (1938).

[10] W. FLÜGGE, Viscoelasticity. Blaisdell (1967).

[11] H. S. CARSLAW and J. C. JAEGER, Conduction of Heat in Solids. Oxford University Press (1948).

[12] S. TIMOSHENKO and J. N. GOODIER, Theory of Elasticity. McGraw-Hill (1951).

[13] E. STERNBERG, Proc. of the Third Symp. on Naval Structural Mechanics (1963).

[14] E. STERNBERG and M. E. GURTIN, Proc. I.V.T A.M., Symp. on Second Order Effects of Elasticity, Plasticity, and Fluid Dynamics (1962).

[15] R. MUKI and E. STERNBERG, J.appl. Mech. 28, 193 (1961).

[16] W. V. LOVITT, Linear Integral Equations. Dover (1950).

[17] T. M. APOSTOL, Mathematical Analysis. Addison-Wesley (1964).

(Received 8 May 1970)

\section{APPENDIX}

The mapping hypothesis, as proposed, contains six adjustable material coefficients, three for creep data and three for relaxation data. For environmental histories which are temporally constant, $\phi(t)=\phi_{p}$, the constitutive equation (2.4) has the Riemann-Stieltjes convolution form discussed in [3] whose one-dimensional form is

$$
\sigma(t)=\int_{0^{-}}^{t} \epsilon(t-\tau) \mathrm{d} G\left(\tau, \phi_{p}\right) .
$$

Let $J\left(t, \phi_{p}\right)$ denote the creep function associated with the above environmental history. Since $J\left(t, \phi_{p}\right)$ is the strain history corresponding to the stress history $\sigma(t)-1(t)$, (A.1) yields the following relationship between associated material response functions

$$
\int_{0^{-}}^{t} J\left(t-\tau, \phi_{p}\right) \mathrm{d} G\left(\tau, \phi_{p}\right)=1(t)
$$

Assume that $G\left(t, \phi_{p}\right)$ has been determined from an experimental program and is given by (3.2). Then (A.2) is a Volterra integral equation of second kind for the associated material response function $J\left(t, \phi_{p}\right)$, which is not necessarily of the form of (3.6). The general solution of equation (A.2), easily obtained by the method of Successive Approximations $\dagger$ for a specific $G\left(t, \phi_{p}\right)$, exists and is unique. For certain special forms of $G\left(t, \phi_{p}\right),($ A.2) gives rise to two useful, simple results.

First, for every fixed $\phi_{p}$ let $G\left(t, \phi_{p}\right)$ and $J\left(t, \phi_{p}\right)$ satisfy (A.2) and let $G\left(t, \phi_{p}\right)$ be given by (3.2). Then the associated material response function $J\left(t, \phi_{p}\right)$ can be written in the form

$$
J\left(t, \phi_{p}\right)=\hat{J}\left[\gamma\left(\phi_{p}\right) t, \phi_{p}\right]
$$

where $\hat{J}$ is a new creep function. To verify this result first note that $1(t)=1\left[\gamma\left(\phi_{p}\right) t\right]$. Then define a relaxation function $\hat{G}$ for a new time variable $\xi=\gamma\left(\phi_{p}\right) t$ by rewriting (3.2) as

$$
\hat{G}\left(\xi, \phi_{p}\right)=G\left(\frac{\xi}{\gamma\left(\phi_{p}\right)}, \phi_{p}\right)=\alpha\left(\phi_{p}\right) 1(\xi)+\beta\left(\phi_{p}\right) G(\xi, \Phi) .
$$

Employing (A.4) and the change of variable theorem $¥$ for Riemann-Stieltjes integrals gives

$$
\int_{0}^{\xi} J\left(\frac{\xi}{\gamma}-\frac{\bar{\xi}}{\gamma}, \phi_{p}\right) \mathrm{d} \hat{G}\left(\bar{\xi}, \phi_{p}\right)=1(\xi)
$$

where $\bar{\xi}=\gamma t$. Next define

$$
J\left(\frac{\xi}{\gamma}, \phi_{p}\right)=\hat{\jmath}\left(\xi, \phi_{p}\right) .
$$

Combining (A.5) and (A.6) and comparing the result with (A.2) we see that $\hat{J}$ is the unique inverse of $\hat{G}$ and the desired result is confirmed.

When the relaxation function is given by (3.2), the associated creep function defined by (A.2) does not have a simple analytic form. In particular, it is different from that in (3.6). For the special case $\alpha=0$ in (3.2), the solution of (A.2) can easily be obtained.

† See Theorem 9.7, reference [17].

$\ddagger$ For example, see reference [16], section 9 . 
Let $\xi=\gamma\left(\phi_{p}\right) t$. Equations (3.2), (A.4) and (A.6) permit (A.5) to be written as

$$
\int_{0^{-}}^{\xi} \hat{J}\left(\xi-\bar{\xi}, \phi_{p}\right) \mathrm{d} \beta\left(\phi_{p}\right) G(\bar{\xi}, \Phi)=1(\xi)
$$

or since $\beta\left(\phi_{p}\right)$ is constant

$$
\int_{0^{-}}^{\xi}\left\{\beta\left(\phi_{p}\right) \hat{J}\left(\xi-\bar{\xi}, \phi_{p}\right)\right\} \mathrm{d} G(\bar{\xi}, \Phi)=I(\xi) .
$$

Hence (A.8) is of form (A.2) which defines the creep function $J(\xi, \Phi)$ associated with $G(\xi, \Phi)$. Since $J(\xi$, $\Phi)$ is uniquely determined by this equation, (A.3) and (A.8) immediately yield

$$
\begin{aligned}
J\left(t, \phi_{p}\right) & =\hat{J}\left[\xi, \phi_{p}\right] \\
& =\frac{1}{\beta\left(\phi_{p}\right)} J\left[\gamma\left(\phi_{p}\right) t ; \Phi\right] .
\end{aligned}
$$

Thus, for the case when $\alpha=0, J\left(t, \phi_{p}\right)$ is given by (3.6) provided

and

$$
\begin{aligned}
& \hat{\alpha}\left(\phi_{p}\right)=0 \\
& \hat{\beta}\left(\phi_{p}\right)=1 / \beta\left(\phi_{p}\right),
\end{aligned}
$$

$$
\hat{\gamma}\left(\phi_{p}\right)=\gamma\left(\phi_{p}\right)
$$

Résumé-Cette étude s'étend sur la théorie linéaire de la thermo-visco-élasticité pour inclure un groupe plus étendu de matériaux et d'effets d'environnement. Le développement s'appuie sur l'observation que pour de nombreux matériaux la courbe de fluage qui résulte d'un essai pour une valeur constante d'un paramètre d'environnement peut être tracée d'après la courbe de fluage pour une autre valeur du paramètre d'environnement: (1) en déplaçant la courbe le long de l'axe logarythmique des temps, (2) en déterminant l'échelle de la réponse élastique initiale, et (3) en déterminant l'échelle de la réponse à long terme ou résiduelle. Un cylindre visco-élastique, incompressible, dépendant de l'environnement et à paroi épaisse est analysé pour un matériau dont on peut créer le modèle à partir du traçage des courbes proposé. Il est trouvé que ce traçage peut avoir un effet prononcé sur la distribution des contraintes résultantes. De plus, en utilisant le traçage ci-dessus, une relation constitutive est développée pour des champs d'environnement transitoires.

Zusammenfassung - Diese Arbeit erweitert die lineare Theorie der Thermo-Viskoelastizität um eine grössere Gruppe von Stoffen und Umgebungswirkungen einzuschliessen. Die Entwicklung beruht auf der Beobachtung, dass für viele Stoffe die Kriechkurve, die aus einer Prüfung bei einem stetigen Wert eines Umgebungsparameters resultiert, auf die Kriechkurve bei einem anderen Wert de; Umgebungsparameters aufgetragen werden kann, durch: (1) Verschiebung der Kurve entlang der logarithmischen Zeitachse, (2) Skalierung des anfangselastischen Ansprechens und (3) Skalierung des langzeitigen oder restlichen Ansprechens. Ein dickwandiger, umgebungsabhängiger, inkompressibler viskoelastischer Zylinder wird für ein Material analysiert, das durch das vorgeschlagene Auftragen modelliert werden kann. Es wird gefunden, dass dieses Auftragen eine profunde Wirkung auf die resultierenden Spannungsverteilungen haben kann. Weiterhin wird unter Benuitzung des gennanten Auftragens eine Materialbeziehung für transiente Umgebungsfelder entwickelt.

Sommario - In questo studio si elabora la teoria lineare della termoviscoelasticità e la si fa includere un gruppo maggiore di materiali ed effetti ambientali. Lo sviluppo poggia sull'osservazione che per molti materiali la curva di scorrimento che risulta da una prova ad un valore uniforme di un parametro ambientale può venire tracciata sulla curva di scorrimento ad un altro valore del parametro ambientale mediante: (1) lo spostamento della curva lungo l'asse di tempo logaritmico, (2) la messa a scala della risposta elastica iniziale, (3) la messa a scala al la risposta residua o a lunga scadenza. Un cilindro viscoelastico incomprimibile a parete spessa dipendente dall'ambiente viene analizzato nel caso di un materiale che può essere modellato mediante la tracciatura proposta. Si scopre che la tracciatura può avere un effetto profondo sulle distribuzioni delle sollecitazioni risultanti. Inoltre, usando la tracciatura di cui sopre si sviluppa un rapporto costitutivo per $\mathrm{i}$ campi ambientali transitori.

Абстракт-Расширена линейная теория термо-вязкоэластичности, чтобы включнть большую группу материалов и зффекты от среды. Разв́итие основано на то, что для,многих материалов криву крипы, 
полученную в одном опытс у одного установившегось значсния парамстра срсды, можно конформно отображить на криве крипы у другого значения параметра среды, если (1) сдвигать криву по логарпфмической оси времены, (2) применить шкалу для начальной эластичной реакции, (3) применить шкалу для долговременной или остаточной реакции. Дается анализ толстостенного, зависящего от среды, несжимаемого, вязкоэластичного цилиндра для одгого материала, который можно отображить при помоши данного метода. Установлено, что отображение можно иметь большое влияние на результирующие распределения напряжения. Кроме того, использование данного отображения позволит развивать конститутвное соотношение для переходных, 'энвиронментальных’ (окружаюших) полей. 